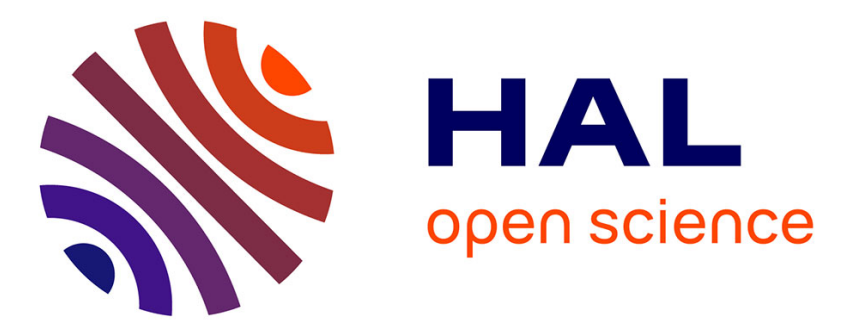

\title{
Le travail bénévole en France et en Europe
}

Edith Archambault

\section{To cite this version:}

Edith Archambault. Le travail bénévole en France et en Europe. Revue française des affaires sociales, 2002, 56 (4), pp.13-39. halshs-00118632

\section{HAL Id: halshs-00118632 \\ https://shs.hal.science/halshs-00118632}

Submitted on 5 Dec 2006

HAL is a multi-disciplinary open access archive for the deposit and dissemination of scientific research documents, whether they are published or not. The documents may come from teaching and research institutions in France or abroad, or from public or private research centers.
L'archive ouverte pluridisciplinaire HAL, est destinée au dépôt et à la diffusion de documents scientifiques de niveau recherche, publiés ou non, émanant des établissements d'enseignement et de recherche français ou étrangers, des laboratoires publics ou privés. 


\section{Le bénévolat en France et en Europe Résumé}

Cet article rappelle les objectifs du programme Johns Hopkins de comparaison internationale du secteur sans but lucratif, qui a servi de cadre aux enquêtes sur le bénévolat en France et en Europe. Avant d'analyser les résultats de ces enquêtes, on s'interroge sur les problèmes méthodologiques rencontrés pour mesurer le bénévolat : définition et délimitation du travail bénévole, degré de fiabilité des résultats d'enquêtes, avantages et inconvénients de la monétarisation du travail bénévole. On analyse ensuite le bénévolat en France : niveau d'engagement, destination du travail bénévole, poids économique du bénévolat, formes d'engagement et caractéristiques socio-démographiques de la population des bénévoles en France. Enfin des données inédites sur le bénévolat dans douze pays d'Europe occidentale et quatre pays d'Europe centrale et orientale sont confrontées avec des données identiques concernant le bénévolat américain afin de dégager les particularités du bénévolat des deux côtés de l'Atlantique. Les données européennes sont ensuite présentées de manière plus qualitative pour dégager quatre variantes de bénévolat européen.

\section{Présentation}

Edith Archambault est professeur de sciences économiques à l'Université de Paris 1 Panthéon-Sorbonne et dirige une équipe de recherche sur le secteur non lucratif au sein de l'Unité mixte de recherche MATISSE (Paris1 et CNRS). Depuis 1990, elle est responsable de la partie française du Programme Johns Hopkins de comparaison internationale du secteur sans but lucratif et elle a participé au comité de pilotage du compte satellite des institutions sans but lucratif de l'ONU. Elle a publié de nombreux articles et une dizaine d'ouvrages dont: Le secteur sans but lucratif. Associations et Fondations en France, Paris , Economica, 1996

\section{Introduction}

Un retour sur les mouvements sociaux du $\mathrm{XX}^{\circ}$ siècle, en France et dans le monde, montre clairement l'impact des bénévoles qui les ont initiés et inscrits dans la durée : du mouvement ouvrier et des suffragettes jusqu'à la protestation contre la mondialisation ultralibérale et au mouvement des « sans » (papiers, droits, travail, logement...) en passant par le pacifisme, le mouvement écologiste, le féminisme, le mouvement des droits civils, l'autoorganisation des minorités de toutes sortes, l'anti-apartheid, l'anti-colonialisme, la théologie de la Libération et Solidarnosc. (Global civil society 2001).

Il y a là de toute évidence une considérable force mondiale d'initiative, de réflexion et de changement social diffusée par une armée d'hommes et de femmes de bonne volonté dont on ne connaît pas les effectifs, faute de statistiques officielles. Le travail rémunéré, en effet, est bien connu grâce aux enquêtes sur les forces de travail qui existent dans de très nombreux pays, parce qu'il contribue à la production au sens des comptes nationaux, au produit intérieur brut. Le travail bénévole, en revanche, ne fait l'objet d'aucune enquête officielle régulière, sauf au Canada, parce qu'il ne contribue pas au PIB. Même le sens de son évolution est incertain: entre ceux qui prophétisent la fin du travail rémunéré et dépendant et l'avènement du travail bénévole et autonome (Rifkin, Gorz) et ceux qui brandissent le spectre du «bowling alone » et dénoncent le déclin de la participation dans les sociétés capitalistes (Putnam), il est impossible de trancher par des données empiriques

Cependant, des travaux expérimentaux de mesure du travail bénévole ont été réalisés, en particulier dans le cadre du Programme Johns Hopkins de comparaison internationale du 
secteur sans but lucratif. Ce programme de recherche a été lancé en 1990, à l'initiative de Lester SALAMON et d'Helmut ANHEIER, professeurs à l'Université Johns Hopkins de Baltimore (Etats-Unis). La première phase de ce programme comparatif a regroupé 13 pays, représentant des niveaux de développement inégaux et des aires culturelles différentes. Les résultats de cette première phase qui s'est déroulée de 1990 à 1995, leur interprétation et la méthodologie sur laquelle ils reposent peuvent être consultés dans Salamon et Anheier (1996) et, pour la France, Archambault (1996). La seconde phase de ce même programme a regroupé 28 pays, dont 24 ont mené les enquêtes sur le bénévolat qui servent de base à cet article.

Les objectifs de ce programme de recherche, identiques pour les deux phases, sont les suivants :

1. Améliorer la connaissance empirique du secteur sans but lucratif que les conventions du système international de comptabilité nationale rendent invisible. Pour ce faire, mesurer le poids, la structure et le financement du secteur sans but lucratif dans un nombre significatif de pays de niveau de développement inégal et appartenant à des aires culturelles différentes.

2. Expliquer par les facteurs historiques, juridiques, politiques et sociaux propres à chaque pays les variabilités observées et identifier, parmi ces facteurs, ceux qui semblent encourager ou retarder le développement du secteur sans but lucratif.

3. Évaluer l'impact que les associations et les fondations ont sur la société et identifier leurs "spécificités méritoires"(Bloch-Lainé, 1995). Le travail bénévole figure évidemment au premier rang de ces spécificités méritoires.

Les résultats obtenus au cours des deux phases de ce programme de recherche ont été jugés vraisemblables par les représentants du milieu associatif dans les divers pays et rapidement repris par les médias et par Bruxelles, en l'absence de toute donnée officielle sur le secteur associatif dans la plupart des pays européens. Pour les pays qui ont participé aux deux phases du programme, comme la France, les résultats obtenus pour les deux années de référence, 1990 et 1995, sont cohérents et marquent une évolution plausible.

Enfin, dans le cadre d'un contrat signé entre l'Université Johns Hopkins et la Commission statistique de l'ONU en avril 1999, un manuel destiné à établir un compte satellite des institutions sans but lucratif a été rédigé, testé sur une douzaine de pays et validé par les instances de l'ONU. Il sera publié dans la série des documents annexes au système international de comptabilité nationale (SNA 93) au printemps 2003 et il est actuellement proposé pour application aux Instituts nationaux de statistique. Ce manuel peut être consulté sur le site internet: http://www.jhu.edu/ gnisp. Comme il comporte une méthodologie de la mesure du travail bénévole, on devrait prochainement sortir de l'ère expérimentale

C'est dans ce contexte que s'inscrivent les résultats qui vont suivre. Leur analyse sera précédée d'une réflexion sur les problèmes méthodologiques rencontrés pour mesurer le bénévolat: définition et délimitation du travail bénévole, degré de fiabilité des résultats d'enquêtes, avantage et inconvénients de la monétarisation du travail bénévole. On analyse ensuite le bénévolat en France: à partir d'une enquête réalisée en 1997 et dont les caractéristiques sont décrites dans l'encadré $\mathrm{n}^{\circ} 1$. Combien y a-t-il de bénévoles et combien de temps travaillent-ils ? pour quels types d'associations? quel est le poids économique du travail bénévole? Quelles sont les caractéristiques de la population des bénévoles et quelles sont ses motivations? Telles sont quelques-unes des questions auxquelles répond cette enquête. Enfin, à partir de données beaucoup moins nombreuses, issues d'enquêtes réalisées 
dans le cadre du programme Johns Hopkins, mais totalement inédites en France et n'ayant fait l'objet que de documents de travail aux Etats-Unis, on caractérisera le bénévolat dans seize pays européens et on le comparera à son homologue américain.

\section{1) Problèmes méthodologiques rencontrés lors de la mesure du bénévolat}

Il n'existe actuellement aucune mesure régulière par l'INSEE du temps de travail bénévole ${ }^{1}$, qu'il soit exercé à l'intérieur des ménages, aboutissant à une production domestique, ou qu'il ait lieu dans un cadre associatif, contribuant ainsi à la production de l'organisation concernée.

Le système international de comptabilité nationale (SCN 93 - SEC 95) actuellement en vigueur retient en effet deux concepts de production. Selon la définition large, la production, c'est "l'ensemble des biens et services susceptibles de d'être vendus sur un marché, ou au moins fournis par une unité à une autre contre rémunération ou gratuitement » (1993 SNA 1.20). Selon cette définition, le travail bénévole est à l'intérieur de la frontière de la production, puisqu'il fournit des produits susceptibles d'être échangés. Cependant, c'est une définition plus restreinte qui est retenue pratiquement par le cadre central des comptes nationaux; elle exclut les services rendus par la travail non rémunéré, qu'il soit domestique ou associatif, alors que la définition large peut être utilisée dans les comptes satellites. Outre l'imprécision de la mesure du travail bénévole, la raison donnée pour cette exclusion est que le travail bénévole n'entre pas dans le champ de la politique économique, usage principal des comptes nationaux(SCN 93, 6.22),

Pour réaliser les trois enquêtes successives sur le bénévolat en France, il a fallu délimiter le travail bénévole, extrapoler les résultats de l'enquête à l'ensemble de la population et enfin donner une valeur monétaire au temps de travail bénévole.

\section{1) La délimitation du travail bénévole.}

Le travail bénévole est un travail, qui doit être distingué du loisir, exercé bénévolement, ce qui le distingue du travail rémunéré.

La distinction entre travail bénévole et loisir repose traditionnellement sur le critère de la tierce personne (Hawrylyshyn, 1977). La personne présumée bénévole peut-elle ou non être remplacée par un salarié ? Si oui, il s'agit d'un travailleur bénévole, si non, d'un simple membre de l'association. On distinguera ainsi ceux qui donnent des leçons de tennis des simples joueurs, ceux qui organisent une réunion ou une manifestation de ceux qui y participent. Le clivage n'est cependant pas toujours aussi net ; c'est le cas quand la qualité de la relation dépend du fait que le travail est bénévole ou quand il s'agit de militantisme. Ainsi, puis-je payer quelqu'un pour visiter à ma place un prisonnier ou un malade ? Ou pour écrire un article dans la presse syndicale ou politique? Ces tâches sont cependant des champs traditionnels du bénévolat où le critère de la tierce personne fonctionne mal. Dans les enquêtes déclaratives, même si des exemples peuvent préciser la délimitation du champ ${ }^{2}$, le

\footnotetext{
${ }^{1}$ Les enquêtes de budget-temps auprès des ménages, effectuées tous les 10 ans environ, permettent de mesurer le temps de travail domestique, régulier et quotidien. Elles sont peu utilisables pour la mesure du travail bénévole associatif qui n'intervient pas nécessairement au cours de la semaine de référence. Cependant Prouteau (1999) en tire des résultats qui vont dans le même sens que ce qui est dit ici..

${ }^{2}$ Les exemples qui suivaient la définition du travail bénévole dans le questionnaire étaient les suivants : « faire du travail de bureau pour une association, un syndicat, encadrer un mouvement de jeunesse ou entrainer un club
} 
travail bénévole demeure ce que la personne entend par là et une confusion entre participation et travail bénévole peut exister.

La distinction entre travail bénévole et travail rémunéré est plus facile du fait de l'existence d'un contrat de travail pour les salariés. L'entrepreneur associatif, celui qui a l'initiative de l'organisation est toujours bénévole, puisqu'il n'y a pas de distribution du profit éventuel. Cependant, il existe des situations intermédiaires entre bénévolat et salariat: remboursement des frais professionnels, rémunération en nature, volontariat (travail qualifié à plein temps rémunéré au salaire minimum, comme c'est la règle dans certaines $\mathrm{ONG}$ ou pour le volontariat civil) ou encore simple acceptation par les salariés d'un salaire inférieur à celui du marché ou d'horaires supérieurs à la norme, parce qu'ils adhèrent aux buts de l'association, ce qui donne à leur travail une dimension bénévole. Là aussi, les enquêtes doivent se fier à la déclaration du répondant pour délimiter le travail bénévole.

\section{2) L'extrapolation des résultats de l'enquête à l'ensemble de la population.}

Les questionnaires des trois enquêtes successives en France, réalisées en 1991, 1994 et1997, sont similaires à ceux qui ont servi dans les 23 autres pays. Ils apportent des informations :

- sur la proportion de personnes déclarant avoir travaillé bénévolement l'année précédente et sur le nombre d'heures de travail bénévole,

- sur les organisations réceptrices, classées par secteur d'activité, selon la nomenclature ICNPO du Programme Johns Hopkins ${ }^{3}$

- $\quad$ sur les caractéristiques socio-démographiques, le niveau de revenu et le degré de pratique religieuse des bénévoles.

On a donc extrapolé à l'ensemble de la population au-dessus de $18 \mathrm{ans}^{4}$ le pourcentage de personnes déclarant avoir travaillé bénévolement dans l'échantillon enquêté, globalement et activité par activité. Ce nombre a été multiplié par le nombre total d'heures de travail bénévole déclaré au cours de l'année précédente. Pour obtenir l'équivalent temps plein, on a divisé par le nombre d'heures de travail correspondant au temps plein des salariés.

Cette extrapolation simpliste suppose évidemment l'exactitude des déclarations des enquêtés. En général, dans les enquêtes auprès des ménages, on observe une sur-déclaration quand le comportement est "socialement désirable ", alors qu'une sous-déclaration est vraisemblable pour les activités irrégulières, et donc facilement oubliées. Dans le cas du bénévolat, les deux effets peuvent se compenser. En ce qui concerne la France, nous avons confronté le bénévolat déclaré par les bénévoles et celui qui a été déclaré par les associations auxquelles il est destiné et trouvé des chiffres du même ordre (Archambault, Tchernonog 1994). En dépit de cette confrontation favorable, les résultats qui vont être exposés sont d'une fiabilité modérée, et les tris croisés effectués reposent en général sur un trop petit nombre d'observations pour être tous significatifs.

sportif distribuer de la nourriture, des vêtements ou d'autres secours, etre pompier ou sauveteur bénévole, nettoyer les espaces naturels, participer bénévolement à un comité, un conseil d'administration, un syndicat d'initiative...

${ }^{3}$ International Classification of Non-Profit Organisations, utilisée ultérieurement, et présentée en détail dans Archambault 1996

${ }^{4} 44565000$ personnes en 1996 


\section{3) La valorisation monétaire du travail bénévole.}

Attribuer une valeur monétaire à un don de temps peut sembler paradoxal, puisque la gratuité est consubstantielle au bénévolat. Cette pratique est cependant de plus en plus recommandée ou imposée par les autorités de tutelle des associations, pour mieux apprécier la part du financement public et des ressources privées dans le budget des associations. À cette préoccupation, le programme Johns Hopkins ajoutait le souci d'évaluer le poids économique réel du secteur sans but lucratif et de mesurer l'apport productif du travail salarié et du travail bénévole.

Dans tous les pays participant au programme Johns Hopkins, le nombre annuel d'heures de travail bénévole a été multiplié par le taux de salaire moyen non agricole, charges sociales comprises. C'est donc une valorisation au coût de remplacement global, conforme au principe de la tierce personne qui a été retenu. D'autres principe de valorisation monétaire auraient pu être retenus (coût d'opportunité, coût de remplacement activité par activité ${ }^{5}$ ), mais leur mise en œuvre supposait des informations sur la qualification des bénévoles et sur le type de tâches effectuées actuellement indisponibles dans la plupart des pays ${ }^{6}$.

Les hypothèses sous-jacentes à la méthode de valorisation au coût de remplacement global sont les suivantes :

- La qualification et la productivité moyenne des travailleurs bénévoles sont identiques à celles des travailleurs salariés. Bénévoles et salariés sont parfaitement substituables,

- si les bénévoles se portaient sur le marché du travail, le taux de salaire resterait identique.

Ces hypothèses sont évidemment discutables; salariés et bénévoles sont plus complémentaires que substituables, et si les bénévoles se portaient sur le marché du travail, le taux de salaire baisserait vraisemblablement. Tous ces problèmes se posent aussi, mais avec davantage d'acuité, pour la mesure du travail domestique (Goldschmidt-Clermont 1993). Le travail bénévole associatif est en effet plus près du marché du travail que le travail domestique et son poids est moins lourd.

Les problèmes méthodologiques rencontrés lors de la mesure du bénévolat ont été similaires en France et dans les autres pays européens; ils doivent être gardés à l'esprit lors de l'examen des résultats qui suivent.

\footnotetext{
${ }^{5}$ cf Fouquet, Chadeau (1981), Archambault, Anheier, Sokolowski(1996)

${ }^{6}$ Seul le Canada dispose de ces données, grâce à une enquête annuelle sur le travail bénévole, analogue à l'enquête emploi.
} 


\section{Méthodologie de l'enquête de 1997 sur les dons et le bénévolat}

(Laboratoire d'économie sociale, Institut de sondages Lavialle, Fondation de France)

A partir d'un questionnaire réalisé par le Laboratoire d'Economie Sociale,et grâce à un financement spécifique de la Fondation de France, l'enquête de terrain a été réalisée par l'Institut de sondages Lavialle en mai 1997

Échantillon national représentatif composé de 2000 personnes âgées de plus de dix-huit ans.

Répartition géographique en neuf régions (Zones d'Études et d'Aménagement du Territoire de l'INSEE) et en cinq catégories d'agglomération.

Quotas imposés aux enquêteurs (calculés par strates région $\mathrm{x}$ habitat) :

- Catégorie socioprofessionnelle : 6 catégories

- Age de l'interviewé(e) : 5 tranches

- Sexe de l'interviewé(e)

- Activité de la femme

Procédure de terrain : toutes les interviews ont été réalisées en face à face au domicile de la personne interrogée.

Remarques sur la taille de l'échantillon : sur 2000 individus interrogés, seuls 438 disent avoir travaillé bénévolement au cours de l'année précédente. Les données portant sur des sous-échantillons, (comme celles relatives aux caractéristiques des bénévoles sont donc à considérer avec prudence.

Remarques sur la qualité des données : la représentativité de l'échantillon sur quota est très bonne Il y a très peu de non-réponses, sauf à la question portant sur les revenus du ménage.

\section{Le bénévolat en France}

En dépit de leur fragilité méthodologique, on présentera les principaux résultats d'une enquête auprès de 2000 personnes de plus de 18 ans, réalisée en 1997 avec le soutien de la Fondation de France (voir encadré 1). Cette enquête, à partir d'un questionnaire et de classifications internationalement comparables, établis dans le cadre du Programme Johns Hopkins, étudie pour la troisième fois en France les comportements de dons et de bénévolat au cours du mois précédent et de l'année précédente. On présentera ici les résultats concernant le travail bénévole, défini comme : " un travail non rémunéré, du temps passé à rendre service à divers groupes ou organisations en dehors de la famille et des amis ». Cette définition, assortie d'un certain nombre d'exemples (voir la note 2), vise à saisir essentiellement le bénévolat associatif.

On verra successivement combien il y a de bénévoles, pour quel type d'associations ils travaillent et quelles tâches ils y accomplissent, avant de mesurer le poids économique du 
bénévolat. On analysera ensuite les principales caractéristiques socio-démographiques de la population des bénévoles avant d'essayer de dégager leurs motivations.

\section{2) Les bénévoles, combien de divisions?}

Près d'un Français sur quatre - 23\% - déclare avoir travaillé bénévolement au cours de l'année 1996. Si l'on extrapole cette proportion à la population adulte, il y aurait environ dix millions de bénévoles en France. C'est évidemment une définition large du bénévolat, puisqu'il suffit d'avoir donné un coup de main à une association au cours de l'année précédente - à l'occasion du téléthon ou d'une vente de charité, par exemple- pour être inclus dans la population des bénévoles.

La durée moyenne du travail bénévole déclarée est de 5 heures par semaine, soit 24 heures par mois, avec une grande dispersion autour de cette moyenne. Nombreux sont ceux qui n'assurent qu'un travail occasionnel, alors que 500000 personnes seulement travaillent au moins à mi-temps et 1500000 au moins à quart de temps. Ce noyau dur du travail bénévole se situe principalement du côté des associations d'action sociale.

Le travail bénévole est régulier, fidèle et exclusif : régulier puisqu'en moyenne, il dure huit mois dans l'année, fidèle puisque $70 \%$ des bénévoles déclarent travailler pour la même association depuis plus de 3 ans et plus de la moitié depuis plus de 5 ans, exclusif puisque $62 \%$ des bénévoles travaillent pour une seule association.

Par rapport aux deux enquêtes précédentes, la proportion de ceux qui déclarent avoir travaillé bénévolement a augmenté régulièrement : $19 \%$ en 1990, $21 \%$ en 1993, $23 \%$ en $1996^{7}$. Entre 1990 et 1996, la durée moyenne hebdomadaire est passée de 4 à 5 heures. Cette progression est d'autant plus remarquable qu'elle contraste avec le discours habituel des responsables associatifs sur l'essoufflement du bénévolat et avec la chute temporaire du nombre des donateurs en 1996, liée principalement au scandale de l'ARC. Le bénévolat est donc bien une activité de proximité, où celui qui s'engage peut vérifier le sérieux de la cause qu'il défend, alors que le don à une association lointaine repose sur la confiance, construction fragile et vulnérable. L'impression d'une crise du bénévolat viendrait essentiellement d'un fort renouvellement de la demande associative : les associations anciennes ont du mal à attirer de nouveaux bénévoles, alors qu'ils affluent vars les associations et les causes nouvelles.

\section{3) Pour qui travaillent les bénévoles?}

Le graphique 1 montre la répartition du travail bénévole par secteur d'activité des associations. On y voit que les associations sportives et de loisirs se taillent la part du lion, puisqu'elles accueillent le tiers du temps de travail bénévole $(33 \%)$ : on retrouve ici les entraîneurs et gestionnaires des clubs sportifs, les animateurs de clubs de loisirs ou de troisième âge. et les très nombreux occasionnels qui transportent des joueurs ou tiennent un stand lors d'une manifestation sportive ou récréative

\section{Graphique 1 Répartition du travail bénévole par domaine (100\% = Durée totale du travail bénévole)}

Source : d'après Archambault et Boumendil, 1997

\footnotetext{
${ }^{7}$ Cette progression pourrait résulter pour une part de la plus grande médiatisation du bénévolat, qui rend ce comportement plus socialement désirable ( cf remarques méthodologiques supra)
} 


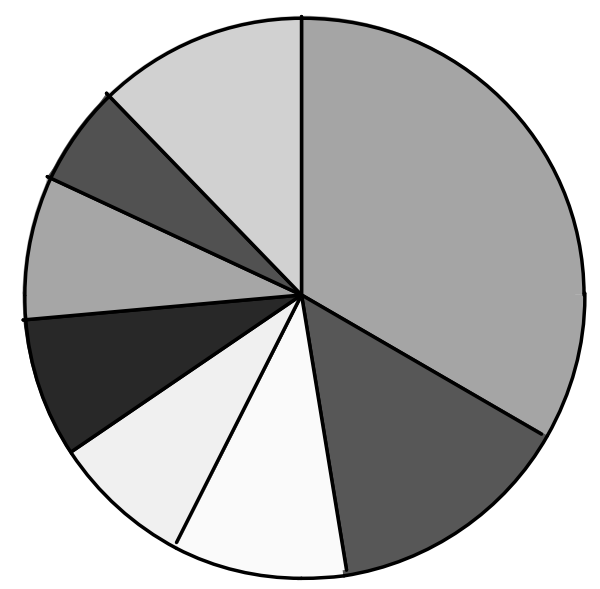

\begin{tabular}{|ll|}
\hline$\square$ sports et loisirs & $\square$ services sociaux \\
$\square$ culture & $\square$ environnement \\
$\square$ religion & $\square$ education \\
$\square$ org. Prof. & $\square$ autres \\
\hline
\end{tabular}

Ensuite, mais loin derrière, arrive le domaine de l'action sociale, avec $14 \%$ du temps de travail bénévole. C'est là que le temps passé par chaque bénévole est le plus long. On y rencontre les bénévoles des associations caritatives, les animateurs de centres sociaux ou de foyers ruraux, les conseillers familiaux ou les permanents des écoutes téléphoniques, les visiteurs de personnes isolées ou en établissement, les tuteurs bénévoles... Une partie de ces tâches de médiation sociale s'est récemment professionnalisée, mais le travail bénévole les a toutes expérimentées préalablement.

Ensuite viennent les associations culturelles, nombreuses et souvent récentes, qui recueillent $10 \%$ de la durée totale de travail bénévole. La plupart de ces associations n'ont pas de salariés et leur existence ne repose donc que sur le bénévolat. Puis, trois secteurs d'activité recueillent chacun $8 \%$ du temps de travail bénévole : les cultes et associations religieuses, avec principalement l'enseignement religieux bénévole, l'éducation et la recherche, avec la montée récente du soutien scolaire, chez les jeunes issus de l'immigration en particulier et, enfin, la défense de l'environnement, domaine où les bénévoles travaillent longtemps et sont souvent très qualifiés. Les associations professionnelles (syndicats, organisations patronales, groupements d'agriculteurs...) ne mobilisent que $6 \%$ du temps de travail bénévole. Enfin, les autres associations dans les secteurs d'activité suivants : santé, aide internationale, logement et développement local, défense des droits et des intérêts ou encore les fondations intermédiaires philanthropiques ne recueillent ensemble que $12 \%$ de la durée totale de travail bénévole.

Le bénévolat en France apparaît donc lié à des activités de sociabilité et d'expression plus qu'à des activités de service ou liées à un projet collectif de société ( Barthélémy 2000, Paugam 1994, Crenner, 1997) 


\section{Tableau 1 Types de tâches remplies par les bénévoles en 1996 et pourcentage de bénévoles déclarant accomplir cette tâche}

\begin{tabular}{|l|c|}
\hline Types de tâches & Pourcentage \\
\hline Participation au conseil d' administration, au bureau, à un comité & 37 \\
Travail de bureau, administratif, gestion, comptabilité & 27 \\
Accueil, écoute téléphonique, conseils, renseignements & 18 \\
Organisation et supervision de manifestations, d'évènements & 46 \\
Soins et visites à domicile & 8 \\
Collecte, distribution, vente de nourriture, vêtements et autres biens & 17 \\
Enseignement, formation & 20 \\
Animation et entraînement sportif & 24 \\
Réparation, construction, aménagement et protection de l'environnement & 10 \\
Premiers secours, lutte contre les incendies et autres catastrophes & 2 \\
Participation à la recherche de fonds, collectes & 11 \\
Autres activités & 7 \\
Ensemble & $\mathbf{2 2 6}$ \\
\hline
\end{tabular}

Source : Archambault et Boumendil, 1997

Que font les bénévoles au sein des associations où ils travaillent ? Ils cumulent en général plusieurs types de tâches, ce qui explique que le total de la colonne du tableau 2 soit très supérieur à $100 \%$. En moyenne, les bénévoles pratiquent un peu plus de deux types d'activité. Cette variété du travail bénévole constitue d'ailleurs un de ses attraits.

La primauté du secteur sportif, culturel et de loisirs explique l'importance des tâches d'organisation de spectacles, fêtes, rencontres, ventes .. qui sont remplies par près d'un bénévole sur deux. Viennent ensuite les activités éducatives: enseignement, formation, animation et entraînement qui concernent $44 \%$ des bénévoles, dont $24 \%$ pour le sport. La participation au conseil d'administration ou à d'autres instances dirigeantes des associations concerne plus du tiers des bénévoles, ce qui fait des associations des lieux d'apprentissage de la démocratie

Le travail administratif ou de gestion, accompli par plus du quart des bénévoles, se retrouve essentiellement dans les petites associations sans salarié. Les autres activités qui occupent 10 à $20 \%$ des personnes déclarant travailler bénévolement sont plus caractéristiques du bénévolat : accueil, écoute et médiation, collecte et distribution de nourriture, protection de l'environnement, secours d'urgence, enfin, collecte de fonds, dont la place dans le travail associatif quotidien est croissante, à l'instar des pays anglo-saxons. Par rapport à l'enquête précédente, on constate cependant une grande stabilité des types d'activités exercées au sein des associations

\section{3) Le poids économique du bénévolat}

Le travail bénévole total représenterait l'équivalent de 1100000 emplois à temps plein ${ }^{8}$, soit près de $5 \%$ des emplois rémunérés en France et une force de travail équivalente à celle des salariés du secteur associatif. Par rapport à 1993, la progression serait de l'ordre de 300000 ETP. C'est évidemment une progression considérable sur une période aussi brève, qui indique

\footnotetext{
${ }^{8}$ Les comparaisons internationales qui suivent ne retiennent pas le bénévolat religieux. Pour la France, le bénévolat est alors de 1010000 ETP
} 
certainement une mutation des comportements quels que soient les biais méthodologiques . La valorisation économique du bénévolat confirme le poids économique du travail gratuit: environ 200 milliards de francs. Si l'on ajoute le bénévolat valorisé, le poids économique du secteur associatif en 1995 passe de 290 milliards de francs (44,2 milliards d'euros) à près de 500 milliards de francs (76,2 milliards d'euros), soit plus de $40 \%$ d'augmentation ${ }^{9}$.

\footnotetext{
${ }^{9}$ On rappelle que ces chiffres expérimentaux résultent du travail réalisé par l'équipe française du Programme Johns Hopkins, phase 2. Il serait souhaitable qu' ils puissent être régulièrement calculés par l'INSEE dans le cadre du compte satellite des Institutions sans but lucratif évoqué en introduction.
} 
Tableau 2 Structure des ressources du secteur sans but lucratif en 1995

\begin{tabular}{|c|c|c|}
\hline $\begin{array}{l}\text { Origine des ressources } \\
\text { valorisé }\end{array}$ & sans bénévolat & avec bénévolat \\
\hline $\begin{array}{l}\text { Financement public } \\
\text { Dons privés } \\
\text { Ressources propres (cotisations, } \\
\text { ventes, participation des usagers...) }\end{array}$ & $\begin{array}{r}58 \% \\
7 \% \\
35 \%\end{array}$ & $\begin{array}{l}33 \% \\
47 \% \\
20 \%\end{array}$ \\
\hline
\end{tabular}

Source : Archambault et Gariazzo 1999

La prise en compte de la valeur économique du bénévolat dans les ressources du secteur associatif change également considérablement la structure de ces ressources ainsi que le montre le tableau 2. Si l'on ne tient compte que des ressources monétaires, le financement public est largement prédominant en France comme dans la plupart des pays européens. Si l'on ajoute aux ressources monétaires la valeur fictive du travail bénévole, les dons deviennent la ressource principale; le don de temps est en effet neuf fois plus important que le don d'argent. Les ressources privées sont alors le double des ressources publiques .

\section{4) Qui sont les bénévoles?}

Contrairement aux idées reçues, les bénévoles sont plus souvent des hommes que des femmes : $25 \%$ des hommes travaillent bénévolement contre $22 \%$ des femmes. Le bénévolat sportif et celui qui s'exerce dans les associations professionnelles expliquent largement cette surreprésentation masculine. Les femmes, en revanche, sont plus nombreuses à s'engager dans l'action sociale.

Graphique 2 Pourcentage de bénévoles suivant l’âge

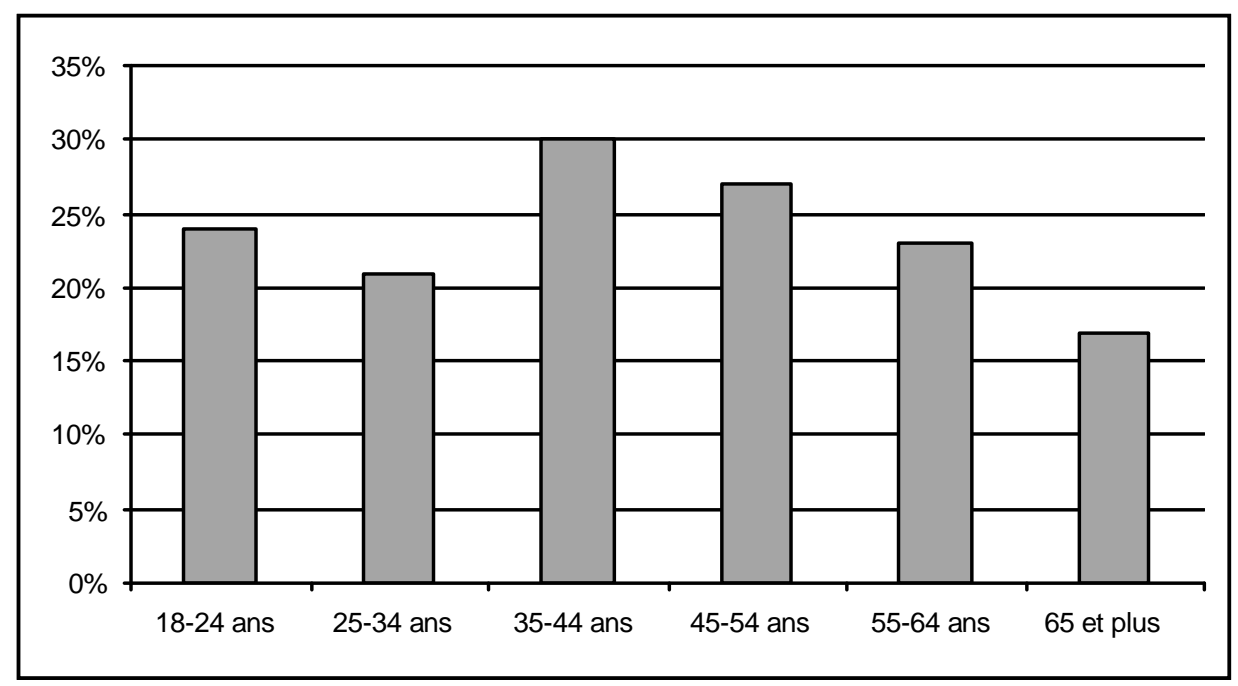

Source : d'après Archambault et Boumendil, 1997

Comme on le voit sur le graphique 2, le bénévolat culmine pour les tranches d'âge de 35 à 54 ans. Il est également lié à la présence au foyer d'adolescents et à une famille nombreuse et concerne alors plus particulièrement les associations de parents d'élèves, les associations 
familiales, les associations culturelles ou sportives et fréquentées par les enfants, où les parents sont sollicités pour l'encadrement. En revanche, le bénévolat des retraités n'est pas très répandu, même s'il est en croissance. Le bénévolat des jeunes lui aussi croît au fil des enquêtes, ce qui va contre les idées reçues et augure favorablement de l'avenir du bénévolat

Tableau 3 Bénévolat selon le niveau d'éducation

\begin{tabular}{|lc|}
\hline Niveau d'éducation & \% de bénévoles en 1996 \\
& \\
Primaire & $14 \%$ \\
Technique ou commercial & $21 \%$ \\
Secondaire & $28 \%$ \\
Supérieur & $31 \%$ \\
\hline
\end{tabular}

Source : Archambault et Boumendil, 1997

L'engagement bénévole croît avec le niveau d'éducation, en France comme dans tous les pays : une personne ayant fait des études secondaires ou supérieures a deux fois plus de chances de travailler bénévolement qu'une personne qui n'a pas dépassé le niveau primaire ainsi que le montre le tableau 4

Tableau 4 Bénévolat selon le revenu mensuel du foyer

\begin{tabular}{|lc}
\hline Revenu mensuel du foyer & \% de bénévoles \\
Moins de 6500F & $16 \%$ \\
$6500-10000 \mathrm{~F}$ & $22 \%$ \\
$10000-15000 \mathrm{~F}$ & $29 \%$ \\
$15000-20000 \mathrm{~F}$ & $28 \%$ \\
Plus de 20 000F (peu significatif) & $(38 \%)$
\end{tabular}

Source : Archambault et Boumendil, 1997

Corrélativement, le bénévolat est sensible au niveau de revenu et croît avec celui-ci, ( cf Tableau 4), ce que confirme l'analyse par catégorie socio-professionnelle: un cadre supérieur a deux fois plus de chances d'être bénévole qu'un ouvrier.

Contrairement à ce que l'on pourrait att endre, le chômage et l'inactivité sont un frein à l'engagement bénévole, lié souvent à l'insertion professionnelle et au réseau de relations qui se noue sur le lieu de travail ( $21 \%$ dans les deux cas). On trouve davantage de bénévoles parmi ceux qui travaillent à plein temps $(25 \%)$, et encore plus parmi ceux -ou surtout celles qui travaillent à temps partiel $(38 \%)$. On pourrait en inférer que la réduction du temps de travail favoriserait le bénévolat, mais pour le moment ce n'est pas démontré : les emplois du temps des salariés passés aux 35 heures semblent privilégier les loisirs, le travail domestique et le temps consacré aux enfants et aux proches plutôt que le bénévolat.

L'appartenance politique et la religion ont peu d'incidence sur le bénévolat, en revanche le degré de pratique religieuse est très influent : plus on est pratiquant, plus on est bénévole : $17 \%$ de bénévoles parmi ceux qui n'ont aucune pratique religieuse, $27 \%$ parmi 
les pratiquants épisodiques, $37 \%$ parmi les pratiquants réguliers. De même, le système de valeurs hérité familialement est très important : ainsi, le bénévolat est deux fois plus fréquent qu'en moyenne quand l'un ou les deux parents de la personne enquêtée ont eux-mêmes été bénévoles .

Enfin l'engagement bénévole est plus fréquent à l'Ouest qu'à l'Est de la France ; il est surtout inversement proportionnel à la taille de l'agglomération où la personne est interrogée. Mais alors que le bénévolat est plus fréquent en zone rurale qu'en zone urbaine, le bénévolat des grandes villes, à Paris en particulier, est plus assidu et régulier, et aussi plus laïc qu'à la campagne

\section{5) Pourquoi s'engage-t-on bénévolement ?}

Sur les motivations profondes du bénévolat, nos enquêtes ne disent rien. Cependant, des entretiens plus qualitatifs que nous-mêmes ou que d'autres ont menés permettent d'émettre quelques hypothèses. Les motivations sont diverses, à la fois collectives et personnelles, altruistes et égoïstes; elles sont certainement différentes selon les secteurs d'engagement bénévole. Ainsi, par exemple, une personne s'engage dans une association de lutte contre une maladie parce que quelqu'un de son entourage a été frappé par cette maladie ; dans une association culturelle ou sportive pour partager et faire connaître une pratique artistique ou sportive qui a été source d'épanouissement personnel, dans une association humanitaire ou caritative pour défendre une cause, lutter contre la guerre ou la misère autrement qu'avec des mots. Ces quelques exemples montrent que le bénévolat part du " goût des autres » et donne du sens à la vie. Mais des motivations plus personnelles existent aussi : s'épanouir, avoir des responsabilités, rencontrer des gens intéressants, acquérir des compétences (les activités associatives figurent de plus en plus sur les curriculum vitae), commencer une carrière politique locale ou nationale...

Plus directement, l'entrée en bénévolat se fait parce qu'une personne de l'association a contacté le candidat ou parce que celui-ci a spontanément offert ses services à l'association. Les intermédiaires philanthropiques, comme le Centre National du Volontariat, sont en effet rares en France et le bénévolat reste donc bien un service de proximité et une insertion dans le milieu local

L'enquête de 1997 cependant interrogeait les personnes n'ayant pas travaillé bénévolement sur les raisons de leur absence d'engagement, avec trois réponses possibles. Parmi les raisons invoquées par ces personnes, l'absence de temps disponible arrive en premier, pour $58 \%$ des non-bénévoles. En second lieu, $40 \%$ préfèrent accorder du temps aux personnes de leur entourage, en troisième position, $20 \%$ disent n'y avoir jamais pensé ou n'avoir jamais été sollicité. Enfin de $5 \%$ à $10 \%$ des non-bénévoles invoquent des raisons de principe : « le bénévolat prend la place du salariat» ou «c'est à l'Etat de prendre en charge les problèmes d'intérêt général »

Comment se situe le bénévolat français par rapport aux autres pays européens ? et peut-on parler d'un modèle européen de bénévolat?

\section{Le bénévolat en Europe}

Les données sur le bénévolat issues de la deuxième phase du Programme Johns Hopkins de comparaison internationale du secteur sans but lucratif portent sur 24 pays. Parmi 
eux, douze appartiennent à l'Europe de l'Ouest (11 pays de l'Union Européenne et la Norvège) et quatre pays d'Europe Centrale et Orientale (Hongrie, République Tchèque, Roumanie, Slovaquie) sont candidats à l'entrée dans l'Union Européenne. Les autres pays ayant conduit des enquêtes sur les dons et le bénévolat sont quatre pays développés: Australie, Etats-Unis, Israel et Japon et cinq pays d'Amérique Latine : Argentine, Brésil, Colombie, Mexique, Pérou.

Nous ne présenterons ici que les résultats pour les douze pays d'Europe occidentale, en les confrontant le plus souvent à l'ensemble des quatre PECO et également aux EtatsUnis qui ont, de loin, la plus importante population de bénévoles. Pour des résultats plus complets et plus détaillés on se reportera à Salamon et Sokolowski 2001 et au site internet : http://www.jhu.edu/cnp/ research.htm. Dans un second temps, le bénévolat en Europe sera présenté de manière plus qualitative dans le cadre des modèles associatifs anglo-saxon, rhénan, scandinave et méditerranéens présentés dans d'autres publications ( Archambault, 1999 et 2001)

2) Le bénévolat en Europe ; analyse quantitative

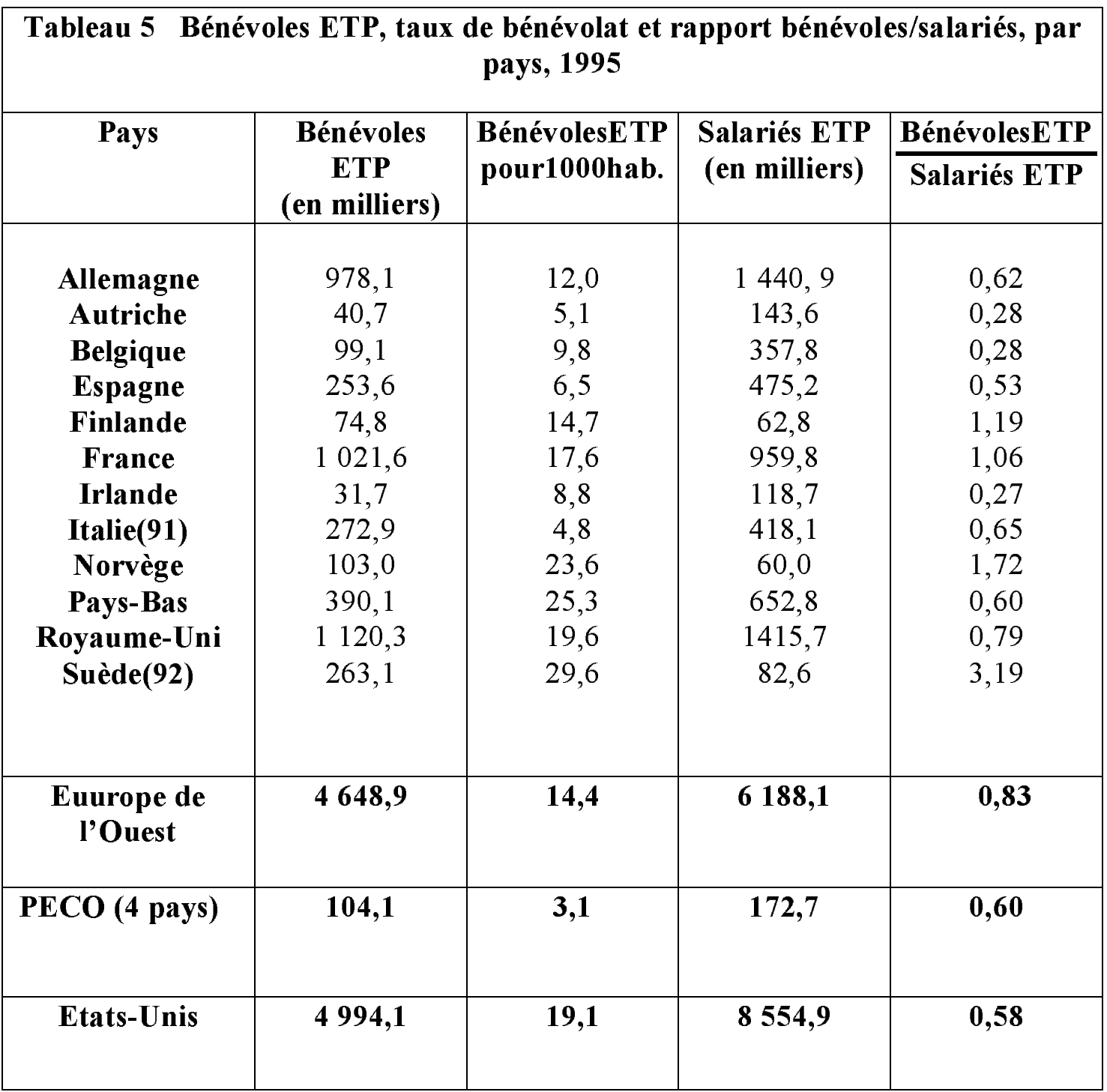

Source : Salamon et Sokolowski, 2001 
Le tableau 5 montre d'abord que le travail bénévole est du même ordre de grandeur aux Etats-Unis et dans l'Union Européenne, environ cinq millions d'emplois ETP ${ }^{10}$. Les taux de bénévolat montrent que ce comportement est très répandu d'abord dans les pays scandinaves et aux Pays-Bas, puis dans les pays anglo-saxons, Royaume-Uni et Etats-Unis, mais la densité du bénévolat est presque aussi élevée en France et en Finlande. Les autres pays sont en dessous de la moyenne européenne (14 bénévoles ETP pour mille habitants). Quant aux PECO, ils ont des taux de bénévolat très bas, liés au caractère récent de leur secteur sans but lucratif, la plupart des associations et fondations étant interdites sous le régime communiste.

On voit aussi clairement, par le rapport bénévoles/salariés, que le secteur sans but lucratif des pays de l'Europe de l'Ouest repose davantage sur le bénévolat que celui des PECO ou des Etats-Unis. Et parmi les pays de l'Europe de l'Ouest, il y a un vif contraste entre des pays où le secteur associatif est très professionnalisé (Irlande, Autriche, Belgique) et d'autres où le bénévolat représente des ressources humaines du même ordre que le salariat (Finlande, France) et encore des pays scandinaves (Suède, Norvège) où le bénévolat l'emporte largement sur le salariat. Pour les PECO, le rapport bénévoles/salariés est du même ordre de grandeur qu'aux Etats-Unis qui ont exporté leur savoir-faire en ce domaine dans les pays en transition.

De même, on pourrait montrer qu'il y a en Europe occidentale une opposition claire entre les secteurs liés à l'Etat-providence, très professionalisés, sans doute parce qu'ils bénéficient d'un financement public en provenance de l'Etat, des collectivités territoriales et de la Sécurité sociale (Education, Santé et dans une moindre mesure Services sociaux) et ceux qui reposent essentiellement sur le bénévolat (Culture, sports et loisirs, Environnement, Défense des droits et des intérêts, aide internationale). Le caractère plus ou moins professionalisé du secteur associatif d'un pays dépend donc pour une part d'un effet de structure; plus l'importance relative des secteurs liés à l'Etat-providence est grande, plus il est professionalisé. Pour une autre part, il dépend d'un mouvement général vers la professionalisation, plus ou moins avancé selon les pays.

Vers quels types d'organisations sans but lucratif se dirigent les bénévoles en Europe occidentale ? C'est ce que l'on peut lire dans le tableau 6 qui compare l'Europe occidentale aux Etats-Unis et aux PECO

\footnotetext{
${ }^{10}$ Les 11 pays de l'UE représentent à peu près $90 \%$ de la population de l'Union Européenne. On peut donc sans risque extrapoler les résultats obtenus à l'ensemble de l'Union Européenne.
} 
Tableau 6 Structure du bénévolat par activité, en \%. Europe occidentale, PECO et Etats-Unis, 1995

\begin{tabular}{|c|c|c|c|}
\hline Activité & $\begin{array}{c}\text { Europe } \\
\text { Occidental }\end{array}$ & PECO & Etats-Unis \\
\hline $\begin{array}{l}\text { Culture, sports et loisirs } \\
\text { Education et recherche } \\
\text { Santé } \\
\text { Services sociaux } \\
\text { Environnement } \\
\text { Développement local, logement } \\
\text { Défense des droits et des intérêts } \\
\text { Fondations intermédiaires philanth ropiques } \\
\text { Aide internationale } \\
\text { Organisations professionnelles } \\
\text { Autres }\end{array}$ & $\begin{array}{c}37,4 \\
6,1 \\
6,5 \\
25,6 \\
3,6 \\
5,2 \\
6,8 \\
0,9 \\
1,9 \\
3,9 \\
2,2\end{array}$ & $\begin{array}{c}34,3 \\
5,4 \\
5,3 \\
28,3 \\
7,7 \\
2,7 \\
5,5 \\
4,0 \\
2,8 \\
3,4 \\
0,4\end{array}$ & $\begin{array}{c}11,8 \\
13,4 \\
13,6 \\
36,7 \\
2,7 \\
0,0 \\
10,2 \\
2,2 \\
0,9 \\
5,5 \\
3,0\end{array}$ \\
\hline
\end{tabular}

Source : Salamon et Sokolowski, 2001

On remarque immédiatement que les bénévoles des PECO s'orientent à peu près vers les mêmes domaines d'activité que leurs homologues occidentaux : culture, sports et loisirs et services sociaux très prioritairement. En revanche, la structure du bénévolat américain est fondamentalement différente : beaucoup moins de bénévolat dans le domaine de la culture et des loisirs, plus commercialisé ; beaucoup plus dans les secteurs de l'Etat-providence, éducation, santé et services sociaux, où l'Etat intervient beaucoup moins qu'en Europe et où les associations et les fondations remplissent des fonctions qui relèvent en Europe principalement du secteur public ${ }^{11}$

\subsection{Le,bénévolat en Europe, analyse qualitative}

Le bénévolat en Europe représente donc une force considérable, du même ordre qu'aux Etats-Unis, mais son orientation est différente. Cependant, l'Europe est diverse et l'on peut distinguer des variantes importantes liées à l'histoire du secteur sans but lucratif et aux relations différentes qu'il entretient avec l'Etat, les collectivités locales et le système de protection sociale, mais aussi avec les Eglises, les syndicats, les partis politiques, les coopératives et les mutuelles. L'importance et la forme du bénévolat dépend aussi du degré de professionalisation des organisations où il s'exerce.

Pour analyser le bénévolat européen plus qualitativement, nous utiliserons les quatre modèles idéal-typiques du secteur sans but lucratif construits à partir des résultats du Programme Johns Hopkins, les modèles rhénan, anglo-saxon, scandinave, et méditerranéen. Ces modèles, présentés ailleurs, recoupent largement la classification d'Esping-Andersen, 1990, des régimes d'Etat-providence ou des colloques MIRE sur la protection sociale en

${ }^{11}$ Pour une comparaison moins sommaire des secteurs sans but lucratif européen et américain, faisant en particulier appel à l'Histoire et aux liens avec les systèmes de valeu $r$ et de protection sociale, voir Archambault, 2001 
Europe. Pour chaque modèle, on caractérisera les organisations sans but lucratif, l'importance et l'orientation du bénévolat et son rôle complémentaire ou substitut au salariat.

\subsubsection{Le modèle rhénan (ou corporatiste)}

Ce type idéal pourrait regrouper l'Allemagne, l'Autriche, la Belgique et les Pays-Bas. Le secteur associatif de ces pays pèse lourd. Il est caractérisé par des organisations puissantes, souvent anciennes, très institutionnalisées et très professionalisées, fédérées selon leur appartenance idéologique, religieuse le plus souvent, mais aussi politique ou syndicale. C'est le système de la "pillarisation", actuellement fortement remis en question. Ces organisations, souvent intégrées dans un système d'État-providence, sont financées essentiellement par l'État et la Sécurité sociale de type bismarckien, conformément au principe de subsidiarité ${ }^{12}$

Le taux de bénévolat dans les pays rhénans est faible en Autriche, moyen en Allemagne et en Belgique et très élevé aux Pays-Bas.. Il s'oriente massivement vers les activités culurelles et de loisirs, et, dans une moindre mesure vers les organisations religieuses et vers l'action sociale, en complémentarité avec les très nombreux salariés des grandes associations de ce secteur ${ }^{13}$. En Belgique plus de la moitié du travail bénévole va vers les associations de service social. Ce bénévolat est comme ailleurs une pratique de proximité, facilitée par la forte tradition de décentralisation des pays rhénans.. La concurrence entre églises catholique et protestante a longtemps marqué le bénévolat dans la plupart des pays rhénans. Ces églises concordataires disposent de moyens importants : en Allemagne, l'État collecte l'impôt pour les églises, qui finance les activités cultuelles mais aussi les services sanitaires et sociaux des réseaux catholique, protestant et juif, ainsi que la formation des bénévoles

\subsubsection{Le modèle anglo-saxon (ou libéral)}

Ce modèle concerne le Royaume-Uni et l'Irlande pour sa variante européenne. Il repose sur des organisations volontaires (voluntary organisations) c'est-à-dire qu'il insiste sur l'importance symbolique du bénévolat, fondée sur une longue tradition individualiste et puritaine d'initiative privée charitable. Cette initiative philanthropique s'exerce en réaction contre l'emprise de l'État fédéral (variante américaine) ou en relation étroite avec les collectivités locales (variante européenne). Au sein de ces organisations, il y a une complémentarité entre des bénévoles nombreux et des salariés très qualifiés. Le niveau de bénévolat est en effet très élevé au Royaume-Uni comme aux Etats-Unis, mais beaucoup moins en Irlande. Les salariés ont souvent suivi des cycles de formation spécialisés orientés vers le secteur non lucratif, ce qui incite les bénévoles à suivre le même type de formation. Il existe une forte culture associative, enracinée dans une longue histoire, transmise par une socialisation précoce au bénévolat à l'intérieur du système scolaire où cette pratique est incluse dans le cursus et valorisée.

Le bénévolat des pays anglo-saxons s'oriente moins qu'en moyenne européenne vers culture, sports et loisirs et davantage vers santé et logement et développement local, cette dernière orientation étant renforcée par le partenariat entre collectivités locales et associations et par la «culture du contrat» qui découle des procédures d'appel d'offres qui se sont multipliées au cours de la dernière décennie (Taylor, 2002). Le bénévolat s'oriente très massivement vers l'action sociale en Irlande (45\% du travail bénévole) alors qu'au Royaume-Uni ce domaine ne recueille que $20 \%$ du bénévolat. Une part importante du

\footnotetext{
${ }^{12}$ selon ce principe, l'Etat ne produit un service collectif que si une unité de moindre taille (région, collectivité locale, association, famille) n'a pas répondu au besoin social. En revanche, il finance.

${ }^{13}$ Caritas, l'équivalent du Secours Catholique, est le premier employeur en Allemagne
} 
bénévolat est liée à de multiples dénominations religieuses concurrentes et à des communautés ethniques relativement fermées, qui souhaitent garder leur identité dans une logique communautariste.

\subsubsection{Le modèle scandinave (ou socio-démocrate)}

Ce modèle regroupe la Suède, la Norvège et la Finlande. En dépit d'une tradition associative ancienne (mouvement ouvrier, ligues de tempérance, organisations religieuses), la plus grande partie du secteur sans but lucratif de ces pays est d'origine récente. Il repose sur des organisations légères et fédérées par champ d'intervention et non en fonction de références idéologiques. L'État providence beveridgien fournit directement les services éducatifs, sanitaires et sociaux et les rares organisations sans but lucratif qui interviennent dans ces domaines comblent les lacunes de l'État providence, selon un modèle de subsidiarité inversée.

La plupart des associations sont donc au service de leurs membres et non de la collectivité ; elles sont très majoritairement gérées par des bénévoles : les associations culturelles, récréatives, sportives (la moitié du travail bénévole) les organisations corporatives et professionnelles ( $15 \%$ du bénévolat), les structures d' accueil des jeunes enfants ou les organisations de consommateurs sont les plus nombreuses. Elles bénéficient d'une participation bénévole très élevée en Suède et en Norvège, un peu moins élevée en Finlande et agissent souvent comme des groupes de pression. Cependant, c'est aussi dans les pays scandinaves que le bénévolat orienté vers la défense des droits de l'Homme ou en faveur de la paix ou de l'aide au Tiers-Monde sont les plus développées. En Finlande, le quart du temps de travail bénévole est destiné à ces causes, non sans lien sans doute avec les accords d'Helsinki et le rôle historique de la Finlande entre l'Est et l'Ouest.

La forte participation syndicale constitue un vivier de bénévoles, car le syndicalisme scandinave fournit des services annexes. L'influence de la religion est inégale selon les pays, mais de plus en plus réduite. Les liens entre l'Etat et le secteur sans but lucratif sont beaucoup plus faibles que dans les autres modèles, encore que la dernière décennie ait vu un mouvement de privatisation de certains services sociaux et les débuts d'une contractualisation qui s'accompagne d'une professionalisation ( Magne et Selle, 2002) 


\subsubsection{Le modèle méditerranéen (ou émergent)}

Les pays qui illustrent ce modèle - Italie, Espagne — ont un secteur associatif moins développé que les précédents, en raison des restrictions importantes, voire des interdictions qui l'ont frappé au cours d'une histoire marquée par les conflits entre l'Église catholique très puissante et l'État et par des périodes de dictature au $\mathrm{XX}^{\circ}$ siècle. C'est aussi un secteur dual, avec d'un côté les organisations charitables liées à l'Église, le réseau scolaire et les services sanitaires et sociaux catholiques et, de l'autre, des organisations professionnelles, syndicales et corporatives, liées au mouvement ouvrier et à un réseau coopératif puissant.

.Le bénévolat associatif est peu développé dans les pays méditerranéens, plus traditionnels et ruraux que ceux d'Europe du Nord, car une entraide informelle, très féminine, s'y exerce encore au niveau de la famille, de la paroisse ou du village. Le bénévolat associatif s'oriente plus qu'en moyenne européenne vers la santé et l'éducation et moins vers les activités culturelles ou récréatives. En Italie le bénévolat d'action sociale est sur-représenté, effet sans doute des coopératives sociales, et c'est le cas de la défense des droits et de l'environnement en Espagne.

\section{Conclusion}

Au terme de cette analyse du bénévolat européen, comment caractériser le bénévolat français ? Par son niveau, il est proche du modèle scandinave ou anglo-saxon. Mais il apparaît moins organisé que dans ces pays et son orientation est proche de celle des pays rhénans. Le bénévolat en France apparaît aussi plus laîc que dans la plupart des autres pays européens qui n'ont pas connu la rupture historique de la Révolution (Archambault 1996). Enfin c'est une pratique plus récente et donc plus fragile que dans les pays d'Europe du Nord qui ont connu plus tôt la liberté associative.

Quelles que soient ses variantes, le bénévolat en Europe représente une puissante force collective, porteuse de valeurs communes et de citoyenneté européenne. Par son orientation prioritaire vers la culture et les loisirs et vers les autres domaines d'expression ou de revendication, le bénévolat européen contraste avec son homologue outre-atlantique, plus tourné vers une fonction de service. Les associations et les fondations, en effet, remplissent actuellement aux Etats-Unis des fonctions qui relèvent en Europe principalement du secteur public, dans le domaine de la santé par exemple, qui représente à lui seul $46 \%$ du secteur sans but lucratif américain, mais aussi dans celui de l'enseignement supérieur où les grandes universités privées prestigieuses recrutent parmi leurs anciens élèves de très nombreux bénévoles chargés du fund-raising, en particulier, mais aussi de nombreuses opérations de communication.

Le bénévolat européen est donc moins gestionnaire qu'aux Etats-Unis, mais plus revendicatif et expressif de l'identité individuelle ou communautaire. Les bénévoles des divers pays européens, en liaison avec les salariés des associations où ils travaillent et avec les chercheurs impliqués dans des programmes européens, ont construit au cours de la dernière décennie des réseaux qui comparent et diffusent les bonnes pratiques (benchmarking). Plus de 100 plate-formes d'associations européennes qui oeuvrent dans le même domaine, sont soucieuses de prendre part à la politique sociale qui commence à s'élaborer à Bruxelles et font du lobbying en ce sens. Ainsi, progressivement s'édifie une culture commune européenne, riche de sa diversité et des échanges auxquels elle donne lieu. L'Europe sociale et l'Europe de la culture ne se construiront pas sans la société civile dont les bénévoles sont l'avant-garde. 


\section{BIBLIOGRAPHIE}

ARCHAMBAULT Édith, (1996) Le secteur sans but lucratif. Associations et Fondations en France, Paris, Economica. ARCHAMBAULT Edith,(1999) "Le secteur associatif en France et dans le monde" in Bloch-Lainé F. Faire Société. Les associations au cour du social, Paris, Syros.

ARCHAMBAULT, E et TCHERNONOG, V. (1994) « Lepoids économique du secteur associatif » RECMA, Revue internationale de l'économie sociale, $\mathrm{n}^{\circ}$ 253-254, p. 118-146

ARCHAMBAULT Édith, BOUMENDIL Judith,(1997) Les dons et le bénévolat en France, Laboratoire d'économie sociale, Fondation de France

. ARCHAMBAULT E. (2001) «Y a-t-il un modèle européen du secteur sans but lucratif? » RECMA, Revue internationale de l'économie sociale, $\mathrm{n}^{\circ} 282$, novembre 2001, p. 64-83

BARTHELEMY M. (2000) Associations : un nouvel âge de la participation, Paris, Presses de Sciences Po

BLOCH-LAINE, F. (1995) "Identifier les associations de service social" RECMA, n²51, 49, p 61-72

BLOCH-LAINE, F. (1999) : Faire Société. Les associations au cour du social, Syros

CREDOC 1999 Les Français et la vie associative Rapport $n^{\circ} 201$.

CREDOC 2001 Le bénévolat au sein des communautés Emmaüs, Etude réalisée à la demande de l'Union Centrale des Communautés Emmaüs, avril 2001

CRENNER E (1997) «Le milieu associatif de 1983 à 1996 »INSEE première, n542

ESPING-ANDERSEN Gosta.(1990) The Three Worlds of Welfare Capitalism. Princeton, Princeton University Press, FERRAND-BEECHMANN D.(1992) Bénévolat et solidarité, Paris, Syros, 1992

Fondation de France (2000) $1^{\text {er }}$ Baromètre de la générosité 1999-2000, octobre 2000

FOUQUET A. et CHADEAU A (1981) «Peut-on mesurer le travail domestique ? Economie et statistique septembre 1981

GASKIN K. AND SMITH J-D A new civic Europe ? A study of the content and role of Volunnteering, London, The Volunteer Centre, 1995

GOLDSCHMIDT-CLERMONT L. (1993) « Monetary valuation of nonmarket productive time. Methodological considerations »The Review of Income and Wealth, $39, n^{\circ} 4$ p.419-433

Global Civil Society 2001, Oxford, Oxford University Press

HAWRYLYSHYN O. (1977) « Towards a definition of nonmarket activities » The Review of Income and Wealth,, $23 n^{\circ} 1$

MAGNE E. et SELLE P. (2002) " A contract culture even in Scandinavia » in ASCOLI et RANCI Eds Dilemmas of the Welfare Mix ; The new structure of welfare in an era of privatisation,-Kluwer-Plenum p.47-76

PAUGAM S. (1994) « L'essor des associations humanitaires » Commentaire, $\mathbf{n}^{\circ} \mathbf{6 1}$

PROUTEAU L. (1999) Analyse économique du bénévolat, Economica, 1999

ROSE-ACKERMAN, S. (1996) « Altruism, nonprofits and economic theory » Journal of Economic Literature vol. 34 701-728

SALAMON L. (1995) Partners in Public Service :Govenment-Nonprofit Relations in the Modern Welfare State, Baltimore, The Johns Hopkins University Press

SALAMON Lester M., ANHEIER Helmut K.,(1996) The Emerging Nonprofit Sector - A Comparative Analysis, Manchester, Manchester University Press,

SALAMON L. ANHEIER H. and associates (1999) Global civil society : Dimensions of the Nonprofit Sector, Baltimore, The Johns Hopkins Center for Civil Society Studies 
SALAMON L and SOKOLOWSKI W (2001)« Volunteering in cross-national perspective : evidence from 24 countries » The Johns Hopkins ComparativeNonprofit Sector Project, working paper $n^{\circ} 40$,

System of National Accounts 1993 , ONU, OECD, FMI, Banque Mondiale, Eurostat

TAYLOR M. (2002) « Government, the Third sector and the contract culture » in ASCOLI and RANCI Eds Dilemmas of the Welfare Mix ; The new structure of welfare in an era of privatisation, Kluwer-Plenum, p. 77-109

TOCQUEVILLE Alexis de (1835) De la Démocratie en Amérique, Paris, Gallimard, 1951 


\section{ANNEXE 2 : CLASSIFICATION INTERNATIONALE DES ORGANISATIONS SANS BUT LUCRATIF (International Classification of Non-Profit Organizations, ICNPO)}

\section{Groupe 1 : CULTURE, SPORTS ET LOISIRS}

1100 - Culture et beaux-arts

Média et communications

Arts plastiques, architecture, céramique

Spectacles (orchestre, théâtre, opéra...)

Musées et conservation du patrimoine

Musique, chorales, groupes musicaux

Groupes artistiques amateurs

Bibliothèques

Ciné-clubs, clubs audiovisuels

Sociétés savantes (historiques, littéraires...)

Centres culturels et artistiques à objet multiple

1200 - Sports et loisirs

Clubs, associations et fédérations sportives (y compris associations de chasse et de pêche)

Clubs de loisirs y compris clubs de $3^{\mathrm{e}}$ âge et amicales diverses (anciens élèves, anciens combattants, conscrits etc...)

Organisations de loisirs à objectif multiple, comités des fêtes

Organisations socio-éducatives et éducation populaire (mouvements de jeunesse),

Rotary, Lyons, Soroptimistes...

1300 - Tourisme social

Maisons et villages de vacances ; colonies de vacances, auberges de jeunesse, gîtes ruraux, campings...

1400 Autres organisations culturelles, sportives et de loisir 


\section{Groupe 2 : ÉDUCATION ET RECHERCHE}

2100 - Enseignement élémentaire, primaire et secondaire

2200 - Enseignement supérieur (au delà du baccalauréat)

2300 - Autres enseignements et formation continue

Écoles professionnelles ou techniques

Formation continue et éducation permanente

Associations périscolaires, associations de parents d'élèves, associations d'étudiants, oeuvres laïques, coopératives scolaires

Organisations éducatives à objet multiple

2400 - Recherche

Recherche médicale

Recherche scientifique et technique

Recherche en sciences sociales et politiques

Organisation de recherche à vocation multiple

2400 - Autres organisations du secteur éducation et recherche

\section{Groupe 3 : SANTÉ}

3100 - Hôpitaux et établissements de rééducation

Hôpitaux généraux

Établissements de rééducation

3200 - Cliniques, maisons de convalescence et maisons de retraite médicalisées

3300 - Santé mentale

Hôpitaux psychiatriques

Soins ambulatoires en santé mentale

Prévention du suicide, centres de désintoxication

3400 - Autres services de santé

Dispensaires, soins infirmier et paramédical

Prévention et éducation sanitaire

Hospitalisation et autres soins à domicile 
Services de rééducation

Services médicaux d'urgence

Associations de malades, de lutte contre une maladie

3500- Autres organisations du domaine sanitaire

\section{Groupe 4 : SERVICES SOCIAUX}

4100 - Établissements et services sociaux

Établissements et services d'accueil et de protection de l'enfance

Établissements et services pour adolescents en difficulté

Établissements et services pour enfants handicapés

Établissements et services pour adultes handicapés (y.c. CAT)

Établissements et services pour adultes en difficulté (Centres d'hébergement et de réhabilitation sociale...)

Services sociaux pour les familles, associations familiales, aide et conseil aux familles

Établissements et services pour personnes âgées (maisons de retraite, établissements de long séjour)

Crèches, garderies, haltes garderies

Aide-ménagère, services de proximité

Services d'entraide et autres services sociaux

Centres sociaux, maisons familiales rurales

4200 - Secours d'urgence et aide aux migrants

Services de sécurité et de secours d'urgence, écoute téléphonique, télé-alarme

Hébergement temporaire

Aide aux réfugiés et aux immigrés

Organisations de secours d'urgence et d'aides aux migrants à objectifs multiples

4300 - Associations caritatives

Secours financiers et autres services aux personnes en difficulté

Secours en nature, distribution de nourriture et de vêtements

Associations caritatives à buts multiples

4400-Autres organisations du domaine social 


\section{Groupe 5 : ENVIRONNEMENT}

Contrôle et réduction de la pollution

Conservation et protection des ressources naturelles

Embellissement des espaces naturels

Protection des animaux

Protection et préservation de la faune sauvage

Services vétérinaires

\section{Groupe 6 : DÉVELOPPEMENT LOCAL ET LOGEMENT}

6100 - Développement économique et local

Associations de voisinage, régies de quartier

Développement économique, pépinières d'entreprises

Syndicats d'initiative

6200 - Logement

Foyers de jeunes travailleurs, d'immigrés

Construction et réhabilitation de logements

Aide au logement

6300- Emploi et formation

Stages d'insertion et de formation professionnelle

Orientation professionnelle, aide à l'emploi, associations de chômeurs

Réinsertion professionnelle : entreprises d'insertion, associations

intermédiaires

6400- Autres organisations de développement local et logement

\section{Groupe 7 : SERVICES JURIDIQUES ET DÉFENSE DES DROITS}

7100 - Associations civique et de défense

Associations civiques

Organisation de défense des minorités, des groupes spécifiques

Défense des libertés publiques et des droits de l'homme

Associations d'étrangers ou d'immigrés

Organisations de protection des consommateurs, des usagers des services publics 
7200 Services juridiques

Assistance juridique, boutiques de droit, centres de gestion

Aide aux victimes

Services juridiques à objectifs multiples

7300- Autres organisations juridiques et de défense des droits

\section{Groupe 8 : INTERMÉDIAIRES PHILANTHROPIQUES ET PROMOTION DU BÉNÉVOLAT}

8100 - Intermédiaires philanthropiques

Fondations recueillant et distribuant des fonds

Promotion et aide au bénévolat

Organisations spécialisées dans la collecte de fonds

8200- Autres organisations intermédiaires philanthropiques et promotion du bénévolat

\section{Groupe 9 : ACTIVITÉS INTERNATIONALES}

9100 - Activités internationales

Relations et échanges culturels internationaux

Associations d'aide au développement

Secours d'urgence international

Défense des droits de l'homme et de la paix dans le monde

\section{Groupe 10 : ASSOCIATIONS PROFESSIONNELLES ET SYNDICATS}

10000 Associations professionnelles et organisations d'agriculteurs; chambres de commerce et de métiers

Ordres et associations de professions libérales

Syndicats

10100- Autres associations professionnelles et syndicales

\section{Groupe 11 : ORGANISATIONS NON DÉNOMMÉES AILLEURS}


